\title{
The evaluation of a communication tool within an acute healthcare organization
}

\author{
Kim Sears ${ }^{1}$, Shannon T. Lewis ${ }^{2}$, Mary Dee M. Craddock ${ }^{2}$, Brandi R. Flowers ${ }^{3}$, Lesley C. Bovie ${ }^{3}$ \\ 1. Queen's University, Ontario, Canada. 2. Regional Municipality of Durham, Ontario, Canada. 3. Lakeridge Health, \\ Oshawa, Ontario, Canada.
}

Correspondence: Kim Sears, RN PHD. Address: Queen's University, School of Nursing, 92 Barrie Street, Room 119, Kingston, Ontario, Canada. E-mail: searsk@queensu.ca

Received: July 15, 2013

DOI : $10.5430 /$ jha.v3n5p79

\section{Abstract}

Background: Striving to ensure clear, concise and consistent communication, the Situation, Background, Assessment and Recommendations (SBAR) tool was adopted for use within a multisite acute healthcare organization.

Objective: To evaluate the use and effectiveness of the SBAR tool on communication within a multisite acute healthcare organization.

Methods: This longitudinal study was conducted over one year and was comprised of four phases including: Phase1) pre-implementation, Phase 2) education sessions, Phase 3) post-implementation, and Phase 4) final evaluation. The study used a convenience sample that included all multidisciplinary participants from Lakeridge Health.

Results: The entire sample in this study consisted of 705 multidisciplinary participants. The number of participants that completed surveys per phase was as follows: phase $1, n=259$, phase $3, n=209$, and phase $4, n=237$. Post implementation of the SBAR tool, participants identified that the tool positively influenced the level of good communication with patients and families. 59.8\% participants felt that good communication existed within their function, $47.4 \%$ identified that good communication existed within their discipline, 45\% recognized that the SBAR tool would work on their units and 53.6\% participants identified that they were familiar with the SBAR tool. During the final evaluation of the SBAR tool, the majority of respondent $66.2 \%$ believed the tool would reduce the potential for errors, $70 \%$ felt that the SBAR process was useful in facilitating communication with other team members or patients, 53.7\% of participants felt communication flow between members of their area or discipline had improved since the implementation of SBAR, 52.1\% of participants felt communication flow between themselves and their colleagues had improved since the implementation of SBAR and $71.7 \%$ of participants identified that they were satisfied when using SBAR that their message was received and understood.

Conclusion: This study is the first study to investigate the use of the SBAR tool across a multisite acute healthcare organization. The tool demonstrated positive results in relation to improving communication with patients and families within the organization. As patients and families are key members of the healthcare team, this finding is important. Although not meeting with a significant change, all of the questions that examined communication, i.e. good communication within function and good interdisciplinary communication, had a positive finding after the implementtation of the SBAR tool. This study examined the importance of leadership to create and sustain a change and the relevance of the culture that people are working within to advance quality within an organization. 


\section{Key words}

Communication, Safety, Acute care, Hospital, Leadership, Culture

\section{I ntroduction}

The purpose of this study was to assess current communication prior to the introduction of the Situation, Background, Assessment, and Recommendation (SBAR) tool and re-assess after the implementation of the SBAR tool at a multisite acute healthcare organization, namely Lakeridge Health. Lakeridge Health is one of the largest community hospitals in Ontario, primarily serving more than 600,000 Durham Region residents. On average, Lakeridge Health's three acute hospitals, one specialty hospital and various off site locations, manage over 540 inpatient beds and deliver care to approximately 1,600 people each day. Over 4,000 staff and physicians along with nearly 1,200 volunteers meet the needs of these patients. One of Lakeridge Health's (LH) strategic directions is to become the safest hospital in Ontario. To help support patient safety and reduce communication-based errors, the senior management team at Lakeridge Health endorsed the organization-wide implementation of the SBAR communication tool. All staff (i.e. all staff, as individuals) was encouraged to participate to determine feasibility of using the tool as a communication wide system.

\section{Review of the literature}

Effective and clear communication is crucial to quality patient care ${ }^{[1]}$, provider safety ${ }^{[2]}$, and organization success ${ }^{[3]}$. A survey of 37 million medical records, revealed that approximately 195000 patients died due to preventable adverse events in 2000, 2001 and $2002{ }^{[4]}$. Over a decade ago in 2002, Chant et al. recognized that the failure to communicate effectively among medical professionals is a major contributor to medical error and poor quality of care ${ }^{[5]}$. In 2004 , the Joint Commission (formerly the Joint Commission on Accreditation of Healthcare Organizations [JCAHO]) completed an analysis of 2455 sentinel events and found the primary cause in over $70 \%$ of cases was communication failure, resulting in the deaths of approximately $75 \%$ of patients involved ${ }^{[6]}$. Further, poor communication has been implicated as a contributing factor in $50 \%$ of hospital administration medication errors and $20 \%$ of adverse drug events ${ }^{\text {[7] }}$. Improving communication has become a priority to advance patient safety and quality of care and was introduced as a National Patient Safety Goal by the Joint Commission in $2003{ }^{[6]}$. Despite this, a 2013 study by Tschanren, et al. revealed that out of a 12-hour shift, registered nurses spent only 42 minutes communicating with other registered nurses, 10.95 minutes communicating with doctors, and only seven minutes communicating with other staff about patient information ${ }^{[8]}$. Communication competence is a cornerstone of organizational effectiveness ${ }^{[9]}$.

SBAR is a communication model that is endorsed by the World Health Organization, the Joint Commission and the Institute for Healthcare Improvement ${ }^{[10,11]}$. SBAR has been shown to improve staff perceptions of the safety culture ${ }^{[6]}$; enhance teamwork ${ }^{[6]}$; ensure that the necessary information is provided during handovers ${ }^{[12]}$; decrease handover time ${ }^{[10]}$; improve medication reconciliation ${ }^{[11]}$; and decrease the frequency of adverse events ${ }^{[11]}$. The SBAR model includes a briefing tool that supports standardized communication ${ }^{[13]}$.

Barriers to communication have been found between nurses and physicians due to differences in communication training ${ }^{[14]}$. Originating in the military, the SBAR tool flattened the hierarchy between junior and senior officers ${ }^{[14]}$. Hierarchy, gender, ethnic background and communication styles also have been found to contribute to barriers in communication. In an attempt to address these common challenges the Joint Commission of Patient Safety identifies that health care organizations must implement a standardized approach to hand over communication ${ }^{[6]}$.

A pilot study conducted at the Toronto Rehabilitation Institute introduced the SBAR communication tool as an intervention ${ }^{[15]}$. Study findings suggest the use of the SBAR tool enhanced interprofessional communication in the rehabilitation context. Overall improvement (greater than $5 \%$ which they considered to be clinically significant) was noted in the areas of: 1) overall perceptions of safety, 2) frequency of events reported, 3) organizational learning-continuous improvement, 4) teamwork within units, 5) feedback and communication about error, 6) staffing, 7) hospital management, 8) support for patient safety, and 9) teamwork across hospital unit. 


\section{Method}

This study was conducted over four phases including: Phase 1) pre-implementation, Phase 2) education sessions, Phase 3) post-implementation, and Phase 4) final evaluation. The study used a convenience sample that included all multidisciplinary participants from Lakeridge Health. The SBAR surveys were adapted from earlier work by Velji et al., (2008) and were used to evaluate the use and effectiveness of the SBAR tool within the organization. It was decided to use these survey tools used in this study as it was noted that the resources from the Toronto Rehabilitation Hospital were open to all to use or modify as needed. The research questions underpinning this study are: 1) Does the SBAR tool improve communication within Lakeridge Health? 2) Do staff identify that they notice a reduction in the potential for the number of errors related to communication? and 3) Do staff acknowledge that they are more satisfied with the quality of communication within their discipline and within the entire health care team? The hypotheses underpinning this study are as follows: 1) using the SBAR communication tool will improve communication within Lakeridge Health; 2) staff will identify that the SBAR tool has reduced the potential for the number of reported errors related to communication and 3) staff will find that the SBAR communication tool provides an opportunity for clear, concise and consistent mechanism of communication within and between various departments and disciplines and with patients.

The study commenced following ethics approval. Descriptive statistics were utilized for quantitative analysis. Survey data from the pre-implementation at time 1 (T1) and the post-implementation time 2 (T2) were compared across the corporation. Following the recommendations from Velji et al. (2008) the results (pre-implementation and postimplementation data) were compared and the results must have shown an improvement of $5 \%$ to be considered better and a drop of $5 \%$ to be considered worse. SPSS statistical software was used.

The surveys were sent out via Survey Monkey and were accessible to all staff over a two-week period. Further, hard copies were made available as not all staff had access to a computer at Lakeridge Health (i.e. Environmental Services). As well, some staff preferred to use a paper-based tool. In this case the data was entered into Survey Monkey by the study coordinator. Results were reported back to the organization as aggregate data.

\subsection{Phase 1: pre-implementation}

The team of investigators met every two weeks over the period of two months to plan the study. The team was primarily composed of nursing staff at various levels within the organization (directors, managers, educators,) the study coordinator, and the primary researcher. An examination of the literature related to communication and the SBAR tool was conducted and various developed tools were examined and presented at one of the early meetings. It was decided that the SBAR assessment tool from Toronto Rehabilitation Center would be modified and used during the pre-implementation phase (see Table 1).

\subsection{Phase 2: educational sessions}

The educational sessions were carefully managed and coordinated as they crossed four sites and numerous patient care units. At the sessions, areas of work and professional delegation of in-serviced staff were tracked to assist in reaching all staff. The sessions were open to all disciplines and positions held within the organization. The sessions were conducted to target audiences throughout the organization. Initially the session started with management and unfolded to include front line staff. The education sessions appeared to be well received by most staff. At the sessions, participants attended a standardized educational in-service on how to use the tool correctly and were provided information regarding how integral standardized communication is to the safety of patients. The educational sessions were 30 minutes in length and were conducted across the sites by the same group of nurse educators to maintain consistency.

\subsection{Phase 3: post-implementation survey}

Three months post-implementation of SBAR, the SBAR assessment tool was re-circulated to provide data that could be compared with the pre-implementation data. The SBAR assessment tool was circulated to the entire organization to 
provide data on the use and impact of the SBAR tool. The SBAR assessment tool (see Table 1) was circulated during a two-week period. The sites were tracked as well as the professions that were engaged in participating in the survey to identify if areas or professions within the corporation still required educational sessions.

Table 1. SBAR assessment tool

Communication questions between functions and disciplines

1. Good communication flow exists between members of your function or discipline?

Strongly Disagree Disagree Neutral Agree Strongly Agree Not Applicable

2. Good communication flow exists between members of the interdisciplinary team or other functions?

Strongly Disagree Disagree Neutral Agree Strongly Agree Not Applicable

3. Good communication flow exists between the health care team and patients and families?

Strongly Disagree $\quad$ Disagree Neutral Agree Strongly Agree $\quad$ Not Applicable

SBAR Questions

4. Are you familiar with the SBAR tool?

Yes (If you answered YES to the above question, please complete the remainder of the survey.) No

5. The SBAR tool will work on your unit?

Strongly Disagree Disagree Neutral Agree Strongly Agree Not Applicable

6. What do you perceive are the challenges with implementing the SBAR tool?

7. If the SBAR communication tool was implemented on your unit would you use it?

Yes

No

\subsection{Phase 4: final evaluation survey}

At the conclusion (one year point) of the study the final evaluation survey tool (see Table 2) was circulated. The surveys were sent out to the entire corporation in the fore mention method. As well, participants had the opportunity to self-identify if they would like to participate in a follow-up focus group.

Table 2. Post SBAR survey

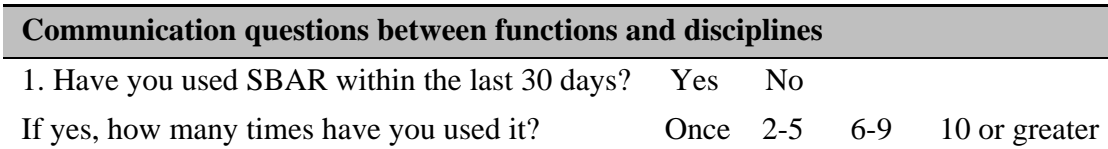

2. Do you believe there is a reduction in the potential for errors related to communication now that SBAR has been introduced?
a. Not at all
b. Slightly
c. Moderately
d. Very Much
e. Significantly

3. Do you feel that the SBAR process was useful in facilitating your communication with other team members or patients?
a. Not at all
b. Slightly
c. Moderately
d. Very Much
e. Significantly

4. Do you feel communication flow between members of your area or discipline has improved since the implementation of SBAR?
a. Not at all
b. Slightly
c. Moderately
d. Very Much
e. Significantly

5. Do you feel communication flow between you and your colleagues has improved since the implementation of SBAR?
a. Not at all
b. Slightly
c. Moderately
d. Very Much
e. Significantly

6. How satisfied are you that when using SBAR your message is received and understood?
a. Not at all
b. Slightly
c. Moderately
d. Very Much
e. Significantly

7. Further evaluation of this tool will include a focus group, if you would like to volunteer to participate in a focus group please identify your name and contact information below.

\section{Results}

The entire sample in this study consisted of 705 multidisciplinary participants. The number of participants per phase was as follows: phase $1, \mathrm{n}=259$, phase $3, \mathrm{n}=209$, and phase $4, \mathrm{n}=237$. 


\subsection{Phase 1: pre-implementation}

During the pre-implementation phase the SBAR Assessment tool (see Table 1) was circulated during the two-week period. In total 259 participants responded the pre-survey (see Table 3). The majority of respondents were allied health 75 (28.9\%) which included radiologists, pharmacists, etc., followed by administration 71 (27.4\%), nurses 51 (19.7\%), clerical 28 (10.8\%), other 27 (10.4\%) which included porters, housekeeping, volunteers, etc. and physicians 7 (2.7\%).

Table 3. Professional participation by phase

\begin{tabular}{llll}
\hline Profession & Phase 1 & Phase 3 & Phase 4 \\
\hline Physician & $7(2.7 \%)$ & $3(1.4 \%)$ & $3(1.3 \%)$ \\
Administration & $71(27.4 \%)$ & $46(22.0 \%)$ & $40(16.9 \%)$ \\
Nurse & $51(19.7 \%)$ & $77(36.8 \%)$ & $79(33.3 \%)$ \\
Allied Health & $75(28.9 \%)$ & $26(12.4 \%)$ & $49(21.1 \%)$ \\
Clerk Clerical & $28(10.8 \%)$ & $30(14.4 \%)$ & $32(13.5 \%)$ \\
Other & $27(10.4 \%)$ & $27(12.9 \%)$ & $34(14.3 \%)$ \\
Total & 259 & 209 & 237 \\
\hline
\end{tabular}

Table 4. Overall results pre (T1) and post (T2) intervention SBAR assessment tool

\begin{tabular}{|c|c|c|c|c|c|c|c|c|}
\hline \multirow{2}{*}{$\begin{array}{l}\text { Item } \\
\text { Time }\end{array}$} & \multicolumn{2}{|c|}{$\begin{array}{l}\text { Strongly Agree } \\
\text { or Agree }\end{array}$} & \multicolumn{2}{|c|}{$\begin{array}{l}\text { Disagree or } \\
\text { Strongly Disagree }\end{array}$} & \multicolumn{2}{|c|}{ Neutral } & \multicolumn{2}{|c|}{ No Response/NA } \\
\hline & $\mathrm{T} 1$ & $\mathrm{~T} 1$ & $\mathrm{~T} 1$ & $\mathrm{~T} 1$ & $\mathrm{~T} 1$ & $\mathrm{~T} 2$ & $\mathrm{~T} 1$ & $\mathrm{~T} 1$ \\
\hline Good Communication Within Function & $59.5 \%$ & $59.8 \%$ & $11.2 \%$ & $15.3 \%$ & $14.7 \%$ & $13.9 \%$ & $14.7 \%$ & $11.0 \%$ \\
\hline Good Communication Interdisciplinary & $44.8 \%$ & $47.4 \%$ & $15.4 \%$ & $17.7 \%$ & $23.9 \%$ & $23.9 \%$ & $15.8 \%$ & $11.0 \%$ \\
\hline Good Communication with Patients and Family & $40.5 \%$ & $46.4 \%$ & $12.4 \%$ & $12.4 \%$ & $22.0 \%$ & $30.1 \%$ & $25.1 \%$ & $11.0 \%$ \\
\hline \multirow[t]{3}{*}{ SBAR Will Work on Unit } & $48.7 \%$ & $45.0 \%$ & $5.4 \%$ & $9.1 \%$ & $25.9 \%$ & $34.9 \%$ & $20.1 \%$ & $11.0 \%$ \\
\hline & \multicolumn{2}{|c|}{ Yes } & \multicolumn{2}{|c|}{ No } & \multicolumn{2}{|c|}{ Blank } & & \\
\hline & $\mathrm{T} 1$ & $\mathrm{~T} 2$ & $\mathrm{~T} 1$ & $\mathrm{~T} 2$ & $\mathrm{~T} 1$ & $\mathrm{~T} 2$ & & \\
\hline Familiar with SBAR & $57.1 \%$ & $53.6 \%$ & $13.5 \%$ & $14.8 \%$ & $29.3 \%$ & $31.6 \%$ & & \\
\hline Would Use if Available & $61.8 \%$ & $56.9 \%$ & $8.9 \%$ & $11.5 \%$ & $29.3 \%$ & $31.6 \%$ & & \\
\hline
\end{tabular}

Of those who responded, 154 (59.5\%) felt that good communication existed within their area; 116 (44.8\%) strongly agreed or agreed that good communication exists within their discipline; and 105 (40.5\%) strongly agreed or agreed that good communication exists with patients and families. 126 (48.7\%) strongly agreed or agreed that the SBAR tool would work on their units and 148 (57.1\%) participants identified that they were familiar with the SBAR tool. In total 160 participants or $61.8 \%$ identified that they would use the SBAR tool if available (see Table 4).

\subsection{Phase 3: post-implementation}

During the post-implementation phase the SBAR Assessment Survey (see Table 1) was re-circulated during the two-week period to assess the effectiveness of the knowledge and use of the SBAR tool. In total, 209 participants responded. The majority of respondents were nurses 77 (36.8\%), followed by administration 46 (22.0\%), clerical 30 (14.4\%), other 27 (12.9\%), allied health 26 (12.4\%), and physicians 3 (1.4\%) (see Table 3). Of those who responded, 125 (59.8\%) participants felt that good communication existed within their function; 99 (47.4\%) strongly agreed or agreed that good communication exists within their discipline; and 97 (46.4\%) strongly agreed or agreed that good communication exists with patients and families. In total 94 (45\%) strongly agreed or agreed that the SBAR tool would work on their units and 112 (53.6\%) participants identified that they were familiar with the SBAR tool (see Table 4). 


\subsection{Comparison of the pre-implementation and post-implementation SBAR assessment survey results}

In the pre-implementation survey 259 participants responded and in the post-implementation survey 209 responded (see Table 5). The majority of respondents in phase 1 were from allied health and in phase 2 were from nursing. The only finding that met with the pre-determined criteria for assessing a positive or negative change ( \pm 5 points) was the SBAR tool appeared to positively influence the level of good communication with patients and families. Although not meeting with a change in \pm 5 , all of the questions that examined communication had a positive change after the implementation of the SBAR tool, including good communication within function (+ 0.35$)$ and good communication interdisciplinary (+ 2.58). Negative results were slightly more statistically significant, but these too failed to reach the 5-point threshold. However, these results showed that there was a negative drop from the pre- intervention and the post-intervention survey in terms of willingness to use the tool -4.84 and familiarity with the tool -3.55 . Additionally a chi-square was run on the data and although a notable effect size was detected the result did not reach significance $(\mathrm{Chi}-\mathrm{Sq}=2.106, \mathrm{df}=5, p$-value $=$ $.834)$.

Table 5. Comparison of pre and post intervention data

\begin{tabular}{|c|c|c|c|}
\hline Item & $\begin{array}{l}\text { Strongly Agree or Agree } \\
\text { Pre-Intervention (\%) } \\
(\mathrm{n}=259)\end{array}$ & $\begin{array}{l}\text { Strongly Agree or Agree } \\
\text { Post-Intervention (\%) } \\
(\mathrm{n}=209)\end{array}$ & Difference (\%) \\
\hline Good Communication Within Function & 59.5 & 59.8 & +0.35 \\
\hline Good Communication Interdisciplinary & 44.8 & 47.4 & +2.58 \\
\hline Good Communication with Patients and Family & 40.5 & 46.4 & +5.87 \\
\hline \multirow[t]{2}{*}{ SBAR Will Work on Unit } & 48.7 & 45.0 & -3.67 \\
\hline & Yes Pre-Intervention (\%) & Yes Post-Intervention (\%) & Difference (\%) \\
\hline Familiar with SBAR & 57.1 & 53.6 & -3.55 \\
\hline Would Use if Available & 61.8 & 56.9 & -4.84 \\
\hline
\end{tabular}

\subsection{Phase 4: evaluation survey}

In phase 4 the evaluation survey (see Table 2) was circulated during the two-week period. In total 237 participants responded. The majority of respondents were nurses 79 (33.3\%), followed by allied health 49 (21.1\%), administration 40 (16.9\%), other 34 (14.3\%), clerical 32 (13.5\%), and physicians 3 (1.3\%) (see Table 3).

Table 6. Results final evaluation survey

\begin{tabular}{|c|c|c|c|c|c|}
\hline Question & Significantly & Very Much & Moderately & Slightly & Not at all \\
\hline $\begin{array}{l}\text { Do you believe there is a reduction in the } \\
\text { potential for errors related to communication } \\
\text { now that SBAR has been introduced? }\end{array}$ & 7 (4.8\%) & 41 (28.3\%) & 48 (33.1\%) & 25 (17.3\%) & $24(16.6 \%)$ \\
\hline $\begin{array}{l}\text { Do you feel that the SBAR process was useful } \\
\text { in facilitating your communication with other } \\
\text { team members or patients? }\end{array}$ & $12(8.2 \%)$ & 55 (37.9\%) & 34 (23.4\%) & 18 (12.4\%) & 26 (17.9\%) \\
\hline $\begin{array}{l}\text { Do you feel communication flow between } \\
\text { members of your area or discipline has } \\
\text { improved since the implementation of SBAR? }\end{array}$ & $5(3.4 \%)$ & 32 (24.1\%) & $41(28.3 \%)$ & 23 (15.9\%) & 44 (30.3\%) \\
\hline $\begin{array}{l}\text { Do you feel communication flow between you } \\
\text { and your colleagues has improved since the } \\
\text { implementation of SBAR? }\end{array}$ & $5(3.5 \%)$ & 34 (23.9\%) & 35 (24.6\%) & 24 (16.9\%) & 44 (31.1\%) \\
\hline $\begin{array}{l}\text { How satisfied are you that when using SBAR } \\
\text { your message is received and understood? }\end{array}$ & $12(8.2 \%)$ & 58 (40\%) & 34 (23.4\%) & 21 (14.5\%) & 20 (13.8\%) \\
\hline
\end{tabular}


When examining the use of the SBAR tool only 202 participants completed this section of the survey, 83 participants (41.1\%) said they had used the tool and 101 respondents (50\%) stated that they had not used the SBAR tool in the last 30 days. When asked if they believed the tool would reduce the potential for errors related to communication 145 participants responded, 96 (66.2\%) of respondents said moderately to significantly. In the study, 101 (70\%) of the 145 participants moderately to significantly felt that the SBAR process was useful in facilitating communication with other team members or patients; and 78 (53.7\%) of participants noted that they moderately to significantly felt communication flow between members of their area or discipline had improved since the implementation of SBAR. 142 people only responded to the next question and 74 (52.1\%) of participants identified that they moderately to significantly felt communication flow between themselves and their colleagues had improved since the implementation of SBAR. 145 participants responded to the final question on the survey and 104 (71.7\%) participants identified that they were moderately to significantly satisfied when using SBAR that their message was received and understood (see Table 6). At the end of the survey there was an opportunity for participants to self-identify if they wanted to be involved in a focus group.

\section{Discussion}

The results demonstrated a positive change in the perception of the level of good communication with patients and their families. This has also been shown to be the case for patients, as after the successful implementation of the SBAR tool, in-hospital patients reported a greater sense of a patient safety culture ${ }^{[17]}$. Although not meeting with a change in \pm 5 , all of the questions that examined communication had a positive change after the implementation of the SBAR tool. These questions included good communication within function $(+0.35)$ and good communication interdisciplinary $(+2.58)$. This finding is consistent with the literature as the SBAR tool has been shown to fundamentally improve the way communication takes place between departments, such as the operating room and recovery, and also during high-risk situations, such as emergencies or handoffs ${ }^{[18]}$. It is also useful in inter professional communication, such as nurse to physician communication or at team meetings where a number of different professionals may be represented ${ }^{[19]}$. Thus, the SBAR tool, when utilized correctly, has been shown to significantly increase patient safety by improving communication between units, professions and during high risk situations, reducing near misses and/or adverse events due to communication errors.

Overall participants strongly agreed that SBAR would work on their unit and they would use it. However, this finding did decrease slightly between the pre-implementation and the post-implementation surveys. It is hypothesized that this slight decrease between phases is attributed to a turnover in senior management, which lead to a change in direction and subsequent reduction in the commitment of senior management to the use of SBAR during the course of this longitudinal study. At the initiation of this study the implementation and evaluation of the SBAR tool was envisioned to be of paramount importance to the organization and was fully supported by the senior management team. Within three months of the initiation of this study there was a significant change in the senior management team and upon completion of the study there had been another change to the senior management team, which may have contributed to the decreased commitment of staff to fully endorse the tool. This change in senior management may have also accounted for the reduction in participants from the administration background as in the first phase of the study there were 77 participants that identified there work function as administrative which reduced to 46 in phase 3 and 40 in phase 4 . Leadership is seen to be an essential aspect to advance quality in healthcare. According to Blouin, in order to develop and sustain an improvement requires committed leadership at all levels within an organization ${ }^{[20]}$.

Another potential rational for the slight decrease in the agreement for the ability of the SBAR tool to work within the organization could be related to the length of time required to evoke a culture change. As this study was conducted over one year, it may not have provided sufficient time to sustain or verify a culture change within the organization. Creating and sustaining a safety culture is seem to be fundamental in the advancement of quality ${ }^{[20]}$. Another challenge that can be faced whenever a new tool or procedure is implemented is the competing time demands of staff as they are often faced with numerous improvements, changes, and surveys on top of the contending demands to deliver high quality patient care. 
Although all aspects of care and practice advancement and research contribute to the advancement of quality there is only so many hours staff have to fulfill their roles.

\subsection{Study limitations}

One limitation of this study was the longitudinal design within the hospital setting as there were significant changes to the composition of the team from inception to completion. This altered the direction of the study at various points, as it required new members of the team to received updating to hold a similar level of comprehension. A second limitation was the support from the leadership team. At the beginning of the study the implementation and use of SBAR was a key initiative, however with changes within the organizational structure, SBAR had been moved from within the leadership groups to being within the staffs' mandate to move forward. Another limitation was that each site within Lakeridge Health holds its own culture as they greatly vary in size and culture with differing communication styles. A final limitation of this study was the reliance on self-reported data. Further, the nature of self-report presents challenges to the validity of the data received; as well, the sensitive nature of the type of data studied presents challenges to the manner in which it is collected $^{[16]}$.

\subsection{Future research directions}

As a next step in the research process the team will be conducting qualitative focus groups from the people who self-identified on the final evaluation surveys that they would be willing to participate. Additionally, it is planned to have yearly assessments conducted using the final evaluation survey tool to re-assess compliance and satisfaction with the SBAR tool.

\subsection{I mplications for practice}

The SBAR tool was shown in this study to assist with communication with patients and families. A key practice implication from this study was the limited awareness of the tool. Although this measure was introduced and numerous educational sessions were given, there remained only 53.9 that strongly agreed or agreed that they were familiar with the SBAR tool upon final evaluation. Respondents that felt they were not aware of the tool were examined closely to identify if there was a site or discipline that could be highlighted. In an attempt to address the limited awareness SBAR is on the orientation agenda for new staff and is envisioned to provide valuable benefits towards sustainability of the SBAR tool. Another practice implication is the challenges of conducting a longitudinal study when there is constant flux within an organization.

The SBAR tool offers benefits to multidisciplinary teams as the way in which communication is taught within educational programs is usually profession specific and yet we expect graduates to communicate together without providing awareness of the differences in communication and ways to overcome these differences. As miscommunication has been shown to be a major cause of error ${ }^{[6]}$ then any advancement in the area of improvement in communication should be further explored and if appropriate utilized. In this study the use of the SBAR tool demonstrated that it was valid for use in a multisite acute healthcare organization.

\section{Conclusions}

This study is the first study to investigate the use of the SBAR tool across a multisite acute healthcare organization. The tool demonstrated positive results in relation to improving communication with patients and families within the organization. As patients and families are key members of the healthcare team this finding is important. Although not meeting with a significant change all of the questions that examined communication i.e. good communication within function and good communication interdisciplinary had a positive finding after the implementation of the SBAR tool. This study examined the importance of leadership to create and sustain a change and the relevance of the culture that people are working within to advance the quality within an organization. 


\section{Competing interests}

This manuscript has not been published and is not under consideration for publication elsewhere. There are no conflicts of interest or competing interests to disclose.

\section{Acknowledgements}

The authors would like to acknowledge Dr. William Goodman for his statistical assistance with the paper and Colleen Peterson for her examination of the final paper.

\section{References}

[1] Agarwal R., Sands, DZ, Schneider JD, Smaltz, DH. Quantifying the economic impact of communication inefficiencies in US hospitals. Journal of Healthcare Management. 2010; 55(4). PMid: 20812527.

[2] Parker-Raley J, Cerroni A, Mottet TP, Lawson KA, Duzinski SV, Mercado M, et al. Investigating pediatric trauma team communication effectiveness phase two: Achieving inter-rater reliability for the Assessment of Pediatric Resuscitation Communication Team Assessment. Journal of Communication in Healthcare. 2013; 6(3): 145-157. http://dx.doi.org/10.1179/1753807612Y.0000000028

[3] McKinley CJ, Perino C. Examining communication competence as a contributing factor in health care workers' job satisfaction and tendency to report errors. Journal of Communication in Healthcare. 2013; 6(3): 158-165. http://dx.doi.org/10.1179/1753807613Y.0000000039

[4] Health Grades. In hospital deaths from medical errors at 195000 per year USA, Medical News Today, 2004.

[5] Available from: http://www.medicalnewstoday. com/articles/11856.php

[6] Chant S, Jenkinson TM, Randle J, Russell G. Communication skills: some problems in nursing education and practice. Journal of clinical nursing. 2012; 11(1): 12-21. http://dx.doi.org/10.1046/j.1365-2702.2002.00553.x

[7] Joint Commission on Accreditation of Healthcare Organizations. Joint commissions perspectives on patient safety. 2005;5(2).

[8] Midlöv P, Bahrani L, Seyfali M, Höglund P, Rickhag E, Eriksson T. The effect of medication reconciliation in elderly patients at hospital discharge. International journal of clinical pharmacy. 2012; 34(1): 113-119. PMid: 22207271. http://dx.doi.org/10.1007/s11096-011-9599-6

[9] Tschannen D, Schoville R, Schmidt P, Buehler K, Borst S, Flaherty-Robb M. Communication practices among nurses in the acute care setting. Journal of Communication in Healthcare. 2013; 6(3): 171-179. http://dx.doi.org/10.1179/1753807613Y.0000000037

[10] Kwantes CT, Boglarsky CA. Perceptions of organizational culture, leadership effectiveness and personal effectiveness across six countries. Journal of International Management. 2007; 13(2): 204-230. http://dx.doi.org/10.1016/j.intman.2007.03.002

[11] Pope BB, Rodzen L, Spross G. Raising the SBAR: how better communication improves patient outcomes. Nursing. 2008; 38(3): 41-43. PMid: 18418180. http://dx.doi.org/10.1097/01.NURSE.0000312625.74434.e8

[12] World Health Organization and Joint Commission. Communication During Patient Handovers. Patient Safety Solutions. 2007; 1(3): 1-4.

[13] Clark E, Squire S, Heyme A, Mickle M, Petrie E. The PACT Project: improving communication at handover. Medical Journal of Australia. 2009; 190(11): S125-S127. PMid: 19485860.

[14] Haig KM, Sutton S, Whittington J. SBAR: a shared mental model for improving communication between clinicians. Joint Commission Journal on Quality \& Patient Safety. 2006; 32(3): 167-175. PMid: 16617948.

[15] Denham C. SBAR for patients. Journal of Patient Safety. 2008; 4(1): 38-48. http://dx.doi.org/10.1097/PTS.0b013e2181660c06

[16] Velji K, Baker GR, Fancott C, Andreoli A, Boaro N, Tardif G, et al. Effectiveness of an adapted SBAR communication tool for a rehabilitation setting. Healthcare Quarterly. 2008; 11(3): 72-79. PMid: 18382165. http://dx.doi.org/10.12927/hcq.2008.19653

[17] Bhandari A, Wagner T. Self-report utilization of health care services: Improving measurement and accuracy. Medical Care Research and Review. 2006; 63(2): 217-235. PMid: 16595412. http://dx.doi.org/10.1177/1077558705285298

[18] Beckett C, Kipnis G. Collaborative Communication: Integrating SBAR to Improve Quality/Patient Safety Outcomes. Journal for Healthcare Quality. 2009; 31(5): 19-28. http://dx.doi.org/10.1111/j.1945-1474.2009.00043.x

[19] Bello J, Quinn P, Horrel P. Maintaining patient safety through innovation: an electronic SBAR communication tool. Computers, Informatics and Nursing. 2011; 29(9): 481. PMid: 21979870. http://dx.doi.org/10.1097/NCN.0b013e31822ea44d

[20] Dunsford J. Structured communication: improving patient safety with SBAR. Nursing for women's health. 2009; 13(5): 384. PMid: 19821914. http://dx.doi.org/10.1111/j.1751-486X.2009.01456.x

[21] Blouin, A.S. High Reliability: Truly Achieving Healthcare Quality and Safety. Frontiers of Health Services Management. 2013; 29(3): 35-40. PMid: 23540044.

Published by Sciedu Press 